\title{
A survey of primary and specialised health care provision to prisons in England and Wales
}

Charles S. Cornford and James Mason School for Health, University of Durham, Queen's Campus, Wolfson Research Institute, Stockton-on-Tees, UK, Katie Buchanan School of Dentistry, University of Manchester, Manchester, UK, David Reeves, Evangelos Kontopantelis and Bonnie Sibbald National Primary Care Research and Development Centre, The University of Manchester, Manchester, UK, Helen Thornton-Jones Department of Public Health and Primary Care, University of Hull, Hull, UK, Mark Williamson HMP Hull and The Quays, Hull PCT and Hull York Medical School, Hull, UK and Lenny Baer Department of Geography, Lancaster University,

Lancaster, UK

\begin{abstract}
Background: Prison health care in England, including primary care, is now incorporated into the National Health Service; the impetus for the change is in part due to concern about standards of health care within prisons. The demographic characteristics and health status of patients within prisons are relatively well understood, as are the problems faced by health care professionals. Less is known about current health care provision. Aims: To describe the organisation of primary health care and specialised services in prisons and compare services available to different types of prison. Method: A piloted questionnaire was sent to the governors of all prisons in England and Wales for completion by the health care manager. Findings: Completed questionnaires were received from 122 (89\%) of 138 prisons. The survey showed a low use of information technology (IT). Problems were reported with the recruitment and retention of general nurses in more than $50 \%$ of prisons. Prisoners in category A/B (higher security) prisons had available to them a greater range of health care services compared with those in other prisons. The results suggest that provision of services for chronic diseases and improvements in IT are needed. Problems with the recruitment and retention of general nurses need addressing. The reasons why lower-security prisoners are receiving a narrower range of specialised health care services compared with higher-security prisoners need justifying.
\end{abstract}

Key words: chronic disease; delivery of health care; prison health; quality of health care

Received: March 2007; accepted: January 2008

\section{Introduction}

Since April 2006 health care services in prisons in England and Wales have become part of the National Health Service (NHS) with general practitioners (GPs) responsible for medical primary care services. The delivery of health care,

Address for correspondence: Charles S. Cornford, School for Health, University of Durham, Queen's Campus, Wolfson Research Institute, University Boulevard, Stockton-on-Tees, TS17 6BH, UK. Email: charles.cornford@durham.ac.uk training for GPs and research in this setting are now therefore explicitly part of general practice as a discipline.

The move to mainstream or normalise care in prisons follows concerns about poor provision of care in prisons (Reed and Lyne, 1997). Policy documents relating to primary care provision within prisons stress two related themes: that patients within prisons should receive the equivalent level of care available outside and that primary care trusts (PCTs) should commission services within prisons (Department of Health 
and HM Prison Service, 2002; Department of Health and Home Office, 2007).

The prison population is currently about 80261 , of which 4370 are female and 12777 are on remand (National Offender Management Service, 2007). Patients in prison are predominately male, young (White et al., 1999) and from areas of high deprivation (Singleton et al., 1998). Although comparisons with similar groups outside prison are not straightforward, patients inside prison are reported to have high consultation rates (Twaddle, 1976), a high prevalence of chronic diseases such as asthma (Butler et al., 2004) and hypertension (Olubodun, 1996), greater prevalence of diseases resulting from illegal drug use such as hepatitis B and C (Butler et al., 1997; Maher et al., 2004; Boutwell et al., 2005) and a higher prevalence of mental health problems (Butler et al., 2005). Older patients have relatively poorer health compared with same-aged groups outside prison, and the prison system is not designed to accommodate their needs (Docherty, 2007). Young offenders have greater physical and psychosocial problems compared with non-offenders of the same age (Macdonald, 2006). Female patients also have relatively poor health and distinctive health needs (Harris et al., 2007). Patients themselves report difficulties accessing outside care, deficiencies in medical care within prisons and fears about dying in prison (Pettinari, 1996). They feel less reassured during consultations than patients outside prisons (Martin et al., 1991).

Difficulties faced by health care staff have also been well described. These include problems concerning truthfulness in consultations (Pettinari, 1996) and working in an organisation where health care is not the main priority (Department of Health, 1999). There is a high turnover of patients (White et al., 1999) and currently, of sentenced prisoners, 5500 are serving sentences of six months or less (National Offender Management Service, 2007). Other problems include deficiencies in care provision outside (Birmingham, 2003), professional isolation (Department of Health and HM Prison Service, 2001) and specific problems such as hunger strikes and dirty protests (smearing of faeces) (Gray et al., 2006). Although there is a need for provision of health-promoting services by nurses in prisons, as well as assessing need and treatment of health care problems, the organisation within prisons may make this difficult to achieve (Condon et al., 2007). The daily routine work of doctors within prisons includes the need to quickly assess large numbers of people newly admitted to prisons, including drug withdrawal symptoms (Marteau and Farrell, 2005).

Less is known about patterns of health care organisation within prisons, particularly primary care services. We undertook, on behalf of the Department of Health, to audit health care provision to prisons and to collect data about the types of prison. Our aims were to describe the nature of services within prisons and to determine what services are associated with which types of prison.

\section{Methods}

A postal survey was conducted in all 138 prisons in England and Wales, including adult and young offender institutions. The survey questionnaire was based on a questionnaire previously used to investigate the quality of care among general practices in England (Campbell et al., 2001), modified to be applicable for prisons by the Primary Care Research Interest Group of the National Prison Health Research Network. Included were questions about the provision of services applicable to prison populations but not usually to mainstream general practice. The full list of these is given in Table 2, and we have termed them, 'specialised services'. The questionnaire gathered information about the number and types of primary health care staff serving in prisons and the organisation of care for five common chronic diseases - diabetes, heart disease, asthma, hepatitis and anxiety/depression and was piloted for acceptability and clarity by health care managers at two prisons.

The questionnaire was sent to all prisons in October 2005. A written reminder was sent after three weeks. Those who did not respond within a further three weeks received one or more telephone reminders.

\section{Statistical analysis}

Individual questionnaire items were analysed descriptively. Other analyses used regression techniques to investigate service provision and health care staff support in relation to prison 
characteristics. These analyses utilised a number of variables constructed as below.

\section{Specialised services provision}

For each prison, provision was measured as the number of specialised services present out of the 13 (Table 2). We excluded mother-and-baby units, as these only applied to women's prisons.

\section{Primary care provision}

We defined a 'full' primary care service for diabetes, heart disease, asthma or hepatitis as one with a patient register, written guidelines and a recall system - features expected of such a service outside of prison. For anxiety/depression we defined a full service to have 'talk therapies' and 'self-help' materials. Each prison was assigned a score, out of 5, based on the number of 'full' chronic care services it provided. In addition, each prison was assigned a score out of 7 , based on the number of 'full' chronic care services it provided plus the existence of on-site out-of-hours care and/ or an on-site pharmacy service (Table 2).

\section{GP support}

Measured as the number of GP surgeries provided per week per 100 prisoners.

\section{Nurse support}

The number of nurse sessions provided per week per 100 prisoners. A 'session' pertains to half-a-day, with a full-time nurse working for 10 sessions a week. In all, $79(65 \%)$ prisons provided reliable data on nurse sessions. We used the mean number of sessions per nurse for this group to impute session numbers for another 36 (30\%) prisons that reported nurse numbers but not sessions per se.

The prison characteristics were:

Size of prison: From inspection of the distribution of prisoner numbers, prisons were divided into three categories of size: small ( $<400$ prisoners); medium (400-699); large (700 plus).

Prison type: We classified the prisons into six types. Adult male prisoners are assigned a security category from ' $A$ ' to ' $D$ ', with 'A' representing the highest risk. We coded these prisons according to the prisoners presenting the highest security risk (remand prisoners are classed as category 'B'). Female prisoners and young offenders are not security classified. We therefore treated women's prisons and young offender institutions as two further distinct categories.

Three sets of analyses were conducted to assess: (1) the influence of prison characteristics (size and type) on the provision of specialised and primary care services; (2) the influence of prison characteristics on GP and nurse staffing levels; and (3) the influence of staffing levels on the provision of chronic disease services, both before and after controlling for prison characteristics. We hypothesised that more staff would result in better organisation of chronic disease services; therefore, this analysis used numbers of GP surgeries and nurse sessions, rather than rates per 100 prisoners.

For outcomes in the form of a count (number of specialised services, number of primary care services, number of chronic services) we applied multivariate Poisson regression. In all cases, the data showed a good fit to the hypothesised Poisson distribution. For staffing support outcomes we applied multivariate linear regression. To examine effects of prison type, we first compared all types, then if this test was significant, performed a sub-test between the four categories of adult male prison.

Prison size was missing in $24(20 \%)$ cases, and prison type in $4(3 \%)$. We dealt with this by treating missing cases as a separate group. This allowed us to include these prisons in the analysis, though we do not report the results for these groups. For simplicity, we excluded the single mixed-sex prison from the regression analysis.

All analyses were conducted using Stata Version 9 (Stata Corporation, College Station, TX, USA) and since this was an exploratory analysis an alpha level of $5 \%$ was used throughout.

\section{Results}

Of the 138 prisons, $122(88 \%)$ responded in time to be included in the analysis. Basic descriptives of the sample are given in Table 1.

\section{Specialised services}

Prisons were asked to indicate from a list which specialised services they had available (Table 2). About half reported an on-site in-patient unit with an average number of 17 beds (range 1-38). 
Table 1 Prison characteristics

\begin{tabular}{ll}
\hline Variables & All prisons $(n=122)$ \\
\hline Prison type & \\
Category $\mathrm{A}^{\mathrm{a}}$ & $8(7)$ \\
Category B $^{\mathrm{ab}}$ & $36(30)$ \\
Category C $^{\mathrm{a}}$ & $31(25)$ \\
${\text { Category } \mathrm{D}^{\mathrm{a}}}_{\text {Women's }}$ & $10(8)$ \\
Young offender & $14(11)$ \\
Missing & $19(16)$ \\
Size (number of prisoners) & $4(3)$ \\
Small (<400) & $37(30)$ \\
Medium (400-699) & $38(31)$ \\
Large ( $\geqslant 700)$ & $23(19)$ \\
Missing & $24(20)$ \\
\hline
\end{tabular}

Figures are number (\%).

a Based on highest security prisoner.

${ }^{b}$ Includes one mixed-gender prison.

Most indicated they had available mental health in-reach team and CARATS (Counselling, Assessment, Referral, Advice and Throughcare Services). Methadone maintenance was offered by just over one-third of prisons. Prisons provided a median of 5 out of 13 specialised services, though the range was very broad with two providing none, and two providing all 13.

\section{Primary care services}

Prisons offered a median of 5 surgeries per week (Table 3), with a minority $(17 \%)$ offering 10. The most common appointment time allocation offered for routine appointments was 'variable' (66\%) followed by '10 min' (26\%), suggesting a greater degree of flexibility in arrangements for numbers of patients seen in a particular surgery and perhaps also reflecting the lack of information technology (IT) (discussed below) compared with outside general practices. GP support was variable, ranging between 0.26 and 4.8 surgeries per week per 100 prisoners, with a mean of 1.6. Nursing support was even more variable; some prisons reported no nursing support and others up to 56 sessions per week per 100 prisoners (mean 14.7). Prisons with no nursing support had all nursing posts vacant at the time of survey.

Out-of-hours care was most commonly organised via an 'in-house' scheme $(30 \%)$, followed by a variety of PCT schemes $(28 \%)$ or a deputising
Table 2 Specialised and primary care services provided

\begin{tabular}{|c|c|}
\hline \multicolumn{2}{|l|}{ Specialised services } \\
\hline In-patient unit & $57(47)$ \\
\hline Mother and baby unit ${ }^{a}$ & $5(33)$ \\
\hline Mental health day care service & $28(23)$ \\
\hline Close supervision unit & $16(13)$ \\
\hline Vulnerable prisoner unit/wing & $38(32)$ \\
\hline Mental health in-reach team & $106(88)$ \\
\hline Medically assisted detoxification & $63(53)$ \\
\hline Methadone maintenance & $43(36)$ \\
\hline CARATS & $114(95)$ \\
\hline Dedicated drug detox unit/wing & $30(25)$ \\
\hline Drug free wing & $50(42)$ \\
\hline Voluntary drug testing wing & $64(53)$ \\
\hline Substance misuse education courses & $76(63)$ \\
\hline Substance misuse day care services & $18(15)$ \\
\hline Median (IQR) specialised services ${ }^{b}$ & $5(3-8)$ \\
\hline \multicolumn{2}{|l|}{ Primary care provision } \\
\hline On-site out-of-hours care & $36(30)$ \\
\hline On-site pharmacy service & $31(25)$ \\
\hline Diabetes service & $70(57)$ \\
\hline Ischemic heart disease service ${ }^{c}$ & $33(27)$ \\
\hline Asthma service ${ }^{c}$ & $64(52)$ \\
\hline Hepatitis service ${ }^{c}$ & $57(47)$ \\
\hline Depression service $^{d}$ & $67(55)$ \\
\hline Median (IQR) primary care services & $3(2-4)$ \\
\hline
\end{tabular}

Figures are number (\%) unless otherwise stated.

$I Q R=$ interquartile range.

${ }^{\text {a }}$ Based on 15 prisons with female prisoners.

${ }^{b}$ Excluding mother and baby units.

${ }^{\mathrm{c}}$ Possesses a patient register, recall system and written guidelines.

Talk therapies and self-help materials available.

service $(20 \%)$. In all, $37 \%$ obtained their pharmacy service from 'another' prison, while $25 \%$ had on-site pharmacists; community pharmacists, hospital pharmacists and others accounted for the remainder.

Only $9 \%$ of prisons described themselves as being 'paper light', with clinical information entered directly onto a computer - a marker of IT use. Almost all prisons could ensure transfer of medical records between prisons, but $73 \%$ had no system for transferring medical records in from the community.

More than a half of all prisons provided full diabetes and asthma services: and nearly half provided a hepatitis service; but only about onequarter had a full service for ischaemic heart disease (Table 2). Only around a third held registers of chronic patients in an electronic form. With the exception of heart disease, around 
Table 3 Organisation of services

\begin{tabular}{lc}
\hline Variables & $5(5-8)$ \\
Median (IQR) GP surgeries per week & $50(23-95)$ \\
Median (IQR) nurse sessions per week & $1.6(0.94)$ \\
Mean (SD) GP surgeries per week per & \\
100 prisoners & $14.7(12.7)$ \\
Mean (SD) nurse sessions per week & \\
per 100 prisoners & \\
Medical records system is 'paper light' & $11 / 120(9)$ \\
Prison has GP registrars & $31 / 110(28)^{\mathrm{a}}$ \\
$\begin{array}{l}\text { Prison has trainee nurses } \\
\text { Prison has a lead for clinical }\end{array}$ & $62 / 117(53)^{\mathrm{a}}$ \\
governance & $117 / 120(98)^{\mathrm{a}}$ \\
$\begin{array}{l}\text { Carried out prisoner health care } \\
\text { satisfaction survey within last }\end{array}$ & $56 / 115(49)^{\mathrm{a}}$ \\
$\begin{array}{l}\text { 18 months } \\
\text { Formal system for dealing with } \\
\text { complaints re health care }\end{array}$ & $117 / 121(97)$ \\
$\begin{array}{l}\text { Formal link with NHS complaints } \\
\text { system }\end{array}$ & $84 / 118(71)$ \\
$\begin{array}{l}\text { Formal meetings to discuss critical } \\
\text { incidents }\end{array}$ & $94 / 122(77)$ \\
$\begin{array}{l}\text { System for obtaining prisoner general } \\
\text { practice medical records }\end{array}$ & $33 / 121(27)$ \\
$\begin{array}{l}\text { System for transferring prison medical } \\
\text { records to another prison }\end{array}$ & $120 / 121(99)$ \\
$\begin{array}{l}\text { System for obtaining medical records } \\
\text { from other prisons }\end{array}$ & $112 / 118(95)$ \\
$\begin{array}{l}\text { Formal mental health training for } \\
\text { discipline staff } \\
\text { Mental health care for prisoners uses } \\
\text { the CPA }\end{array}$ & $101 / 113(89)$ \\
\hline
\end{tabular}

Figures are number/denominator (\%) unless stated otherwise, denominators vary due to missing values. $\mathrm{IQR}=$ interquartile range; $\mathrm{GP}=$ general practitioner; $\mathrm{NHS}=$ National Health Service; $\mathrm{CPA}=$ care programme approach.

a 'Don't know' responses excluded.

two-thirds of prisons had a designated lead for each chronic condition, usually a nurse, and about a half held special clinics. Less than a quarter of prisons had audited any of their chronic disease services in the last two years.

More than half provided both talk therapies and self-help materials for patients with anxiety or depression (Table 2). Talk therapies were delivered by a mixture of providers such as Community Psychiatric Nurses, psychologists and counsellors.

\section{Vacancies}

Although there were a large variety of different staff vacancies and eight prisons had vacancies for GPs, the major difficulty appeared to be with general nurses. In all, $64(52 \%)$ prisons were looking for one or more general nurses to work full or part time. Across all prisons, there were a total of 200 vacancies for nurses.

\section{Arrangements for governance and complaints}

Of the 115 prisons who knew whether or not they had carried out a recent satisfaction survey, $56(49 \%)$ had done so. Almost all stated they had leads for clinical governance and most said they had a formal system for dealing with complaints. About $80 \%$ stated they had formal meetings to discuss critical events.

\section{Factors associated with service provision}

\section{Number of specialised services}

In multivariate regression, specialised service provision was related to both prison type $(P<0.001)$ and prison size $(P=0.007)$. The differences between category A to D prisons alone were also significant $(P<0.001)$ (Table 4$)$. Figure 1 shows the adjusted mean numbers of specialised services for each type and size of prison. Categories A and B and women's prisons provided the widest variety of specialised services, on average around twice as many services as categories $\mathrm{C}$ and D. Medium-sized prisons had on average around $30 \%(10-60 \%)$ more specialised services, and large prisons $50 \%(20-100 \%)$ more, than small prisons.

\section{Number of primary care services}

There was no evidence that provision of primary care services was in any way influenced by prison characteristics.

\section{Factors associated with level of GP and nurse support}

GP support was highest at women's prisons and lowest at category $\mathrm{C}$ prisons $(P<0.001)$, with averages of $2.4(2.0-2.8)$ and $1.2(0.9-1.4)$ surgeries per week per 100 prisoners, respectively (Figure 2 and Table 5). Differences between category A to D prisons were non-significant $(P=0.076)$. Small prisons held more surgeries pro rata than medium or large prisons $(P<0.001)$. Nurse support was also highest at women's prisons $(P<0.001)$, by a wide margin, and slightly elevated at category A and B compared with category C and D prisons 
Table 4 Summary of Poisson regression of service provision on prison characteristics

\begin{tabular}{|c|c|c|c|c|}
\hline \multirow[t]{2}{*}{ Variable } & \multicolumn{2}{|c|}{ Number of specialised services $(n=121)$} & \multicolumn{2}{|c|}{ Number of primary care services $(n=121)$} \\
\hline & IRR & $P$ value & IRR & $P$ value \\
\hline \multicolumn{5}{|l|}{ Prison type } \\
\hline A & $0.94(0.72-1.3)$ & $<0.001(<0.001)^{\mathrm{b}}$ & $1.2(0.81-1.8)$ & 0.708 \\
\hline $\mathrm{B}^{\mathrm{a}}$ & & & - & \\
\hline C & $0.53(0.42-0.65)$ & & $0.98(0.73-1.3)$ & \\
\hline $\mathrm{D}$ & $0.47(0.32-0.68)$ & & $0.77(0.48-1.2)$ & \\
\hline Women's & $0.89(0.68-1.2)$ & & $0.99(0.66-1.5)$ & \\
\hline Young offender & $0.76(0.59-0.96)$ & & $0.82(0.57-1.2)$ & \\
\hline \multicolumn{5}{|l|}{ Prison size } \\
\hline Small ${ }^{\mathrm{a}}$ & - & 0.007 & - & 0.291 \\
\hline Medium & $1.3(1.1-1.6)$ & & $0.97(0.72-1.3)$ & \\
\hline Large & $1.5(1.2-2.0)$ & & $1.3(0.93-1.8)$ & \\
\hline
\end{tabular}

IRR = incident rate ratio.

${ }^{a}$ Reference group.

${ }^{\mathrm{b}}$ Men's prisons (A to D) only.

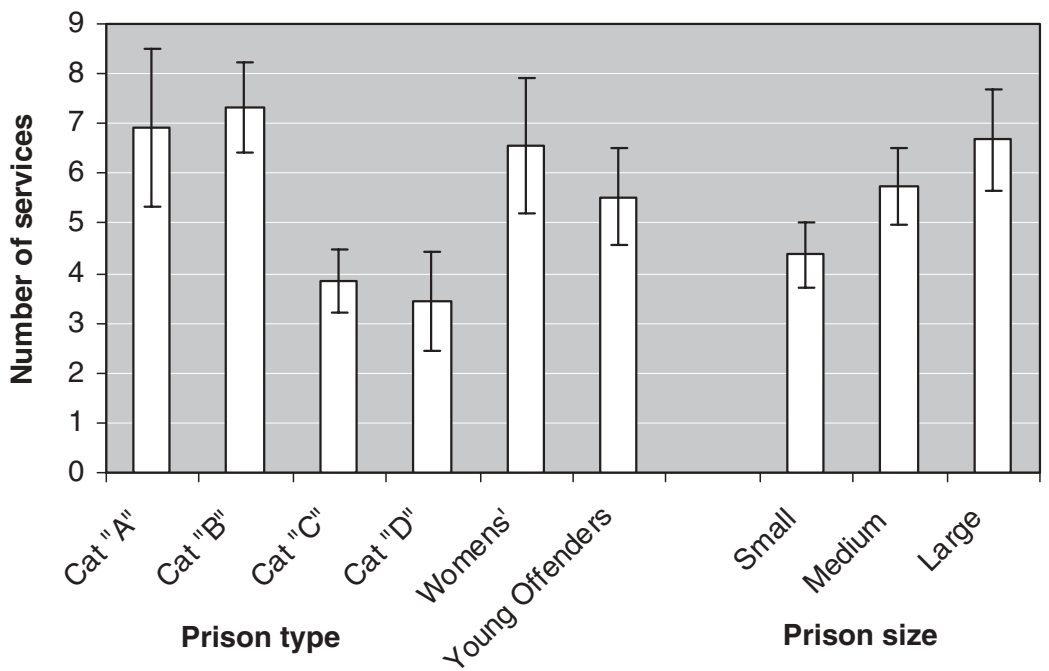

Figure 1 Mean number of specialised services (and 95\% error bars), by prison type (adjusted for prison size) and prison size (adjusted for type)

$(P=0.004)$. Nursing support, like GP support, was lower at medium and large prisons $(P=0.039)$.

\section{Factors associated with chronic disease care}

Provision of chronic disease services was not associated with either GP or nurse staffing levels either before $(P=0.124$ and $P=0.199$, respectively) or after $(P=0.086$ and $P=0.285)$ adjustment for prison characteristics.

\section{Discussion}

\section{Implications}

The use of IT, including electronic records, was low. IT facilitates the structured care necessary for high-quality chronic disease management (Balas et al., 2000; Bodenheimer et al., 2002; Weingarten et al., 2002). Lack of IT potentially excludes prisoners from receiving an equivalent

Primary Health Care Research \& Development 2008; 9: 126-135 

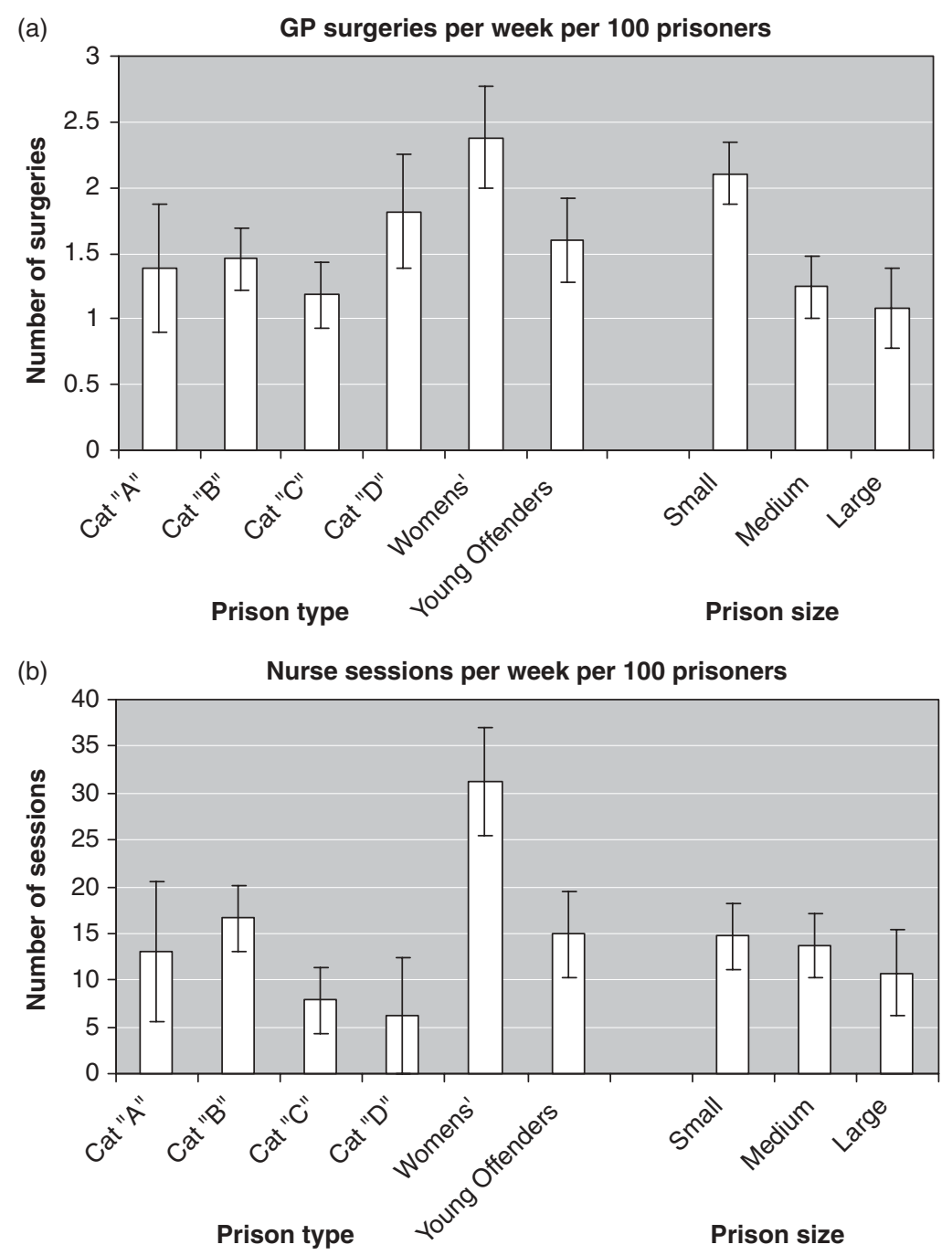

Figure 2 Health care support (and 95\% error bars) by prison type (adjusted for prison size) and prison size (adjusted for type). (a) Mean GP surgeries per week per 100 prisoners; (b) mean nurse sessions per week per 100 prisoners

level of care compared to patients outside. The absence of systems for obtaining medical records from general practices outside is also concerning.

We chose fairly minimal standards to define a 'full' service for chronic diseases. Although we lack hard evidence, we would expect almost all practices outside prison to meet these criteria, whereas substantial numbers of prisons did not. The service was particularly poorly developed for heart disease. This may reflect the absence of patients with heart disease in some prisons, though all are likely to have some patients at some time. Although there are known problems with high prisoner turnover rates (White et al., 1999), high-quality primary care depends on good recall systems for diabetes, heart disease and asthma. Even for diabetes, where the practice of regular recall is well known and in which the benefits of structured care have been established 
Table 5 Summary of multiple regression of health care staff support on prison characteristics

\begin{tabular}{|c|c|c|c|c|}
\hline \multirow[t]{2}{*}{ Variable } & \multicolumn{2}{|c|}{$\begin{array}{l}\text { Number of GP surgeries per week per } 100 \\
(n=121)\end{array}$} & \multicolumn{2}{|c|}{$\begin{array}{l}\text { Number of nurse sessions per week per } 100 \\
(n=114)\end{array}$} \\
\hline & Coefficient & $P$ value & Coefficient & $P$ value \\
\hline \multicolumn{5}{|l|}{ Prison type } \\
\hline A & $-0.07(-0.61$ to 0.46$)$ & $<0.001(0.076)^{\mathrm{b}}$ & $-3.6(-11.6$ to 4.5$)$ & $<0.001(0.004)^{\mathrm{b}}$ \\
\hline $\mathrm{B}^{\mathrm{a}}$ & - & & - & \\
\hline $\mathrm{C}$ & $-0.27(-0.62$ to 0.07$)$ & & $-8.8(-13.8$ to -3.7$)$ & \\
\hline $\mathrm{D}$ & $0.36(-0.14$ to 0.87$)$ & & $-10.4(-18.0$ to -2.8$)$ & \\
\hline Women's & $0.93(0.45$ to 1.4$)$ & & 14.6 (7.6 to 4.3$)$ & \\
\hline Young offender & $0.14(-0.26$ to 0.55$)$ & & $-1.7(-7.7$ to 4.3$)$ & \\
\hline \multicolumn{5}{|l|}{ Prison size } \\
\hline Small ${ }^{\mathrm{a}}$ & - & $<0.001$ & - & 0.039 \\
\hline Medium & $-0.87(-1.2$ to -0.53$)$ & & $-0.90(-5.9$ to 4.1$)$ & \\
\hline Large & $-1.0(-1.4$ to -0.63$)$ & & $-3.9(-10.0$ to 2.1$)$ & \\
\hline
\end{tabular}

${ }^{\text {a }}$ Reference group.

${ }^{\mathrm{b}}$ Men's prisons (A to D) only.

in the prison context itself (MacFarlane et al., 1992), one-quarter of prisons had no recall system.

The low use of methadone maintenance therapy is worrying, given the known benefits including reduction in mortality rates and incarceration rates (Farrell et al., 1994), and needs addressing. The high level of mental health problems in prisoners (Birmingham, 2003) makes the absence of talk therapies in a third of the prisons surprising. The cost of transporting prisoners to community-based facilities for treatment is generally prohibitively high (Department of Health and HM Prison Service, 2006); hence, patients in prisons without on-site services are likely to have no access at all.

Over $50 \%$ of prisons reported one or more vacancies for general nurses. While some prisons might have been actively recruiting new nurses, for example, in connection with moving provision from the prison service to the NHS, this would not fully explain why general nurse vacancies were higher than those for other staff. It may be that there are particular difficulties with recruitment or retention, or both, of general nurses. General practices outside prisons rely increasingly on nurse-led care provision (Woodroffe, 2006); the shortage of nurses in prisons is likely to impact significantly on what is achievable in prisons.

Not all differences between community and prison indicated worse prison health care. Many prisons had systems in place to deal with the management of hepatitis, which would be rare in general practice outside prison, and this may indicate appropriate targeting of services to patient need. The variety of schemes to provide out-of-hours care and pharmacy provision may reflect the need for local pragmatic arrangements in these areas.

Compared with small prisons, medium and larger prisons had a wider range of specialised services. The range of primary care services provided was similar across prisons, although GP and nurse support, relative to prisoner numbers, were lower in large- and medium-sized prisons than smaller ones. There may be economies of scale whereby the levels of staffing required to meet the need reduces as prisoner numbers increase.

Most prisons hold adult males, who are categorised by security rating. Prisoner security rating was related to specialised services but did not appear to influence provision of primary care services. Category A and B prisons provided the widest range of specialised services, considerably more than categories $\mathrm{C}$ and $\mathrm{D}$, although only marginally more than women's prisons. Many of the specialised services address mental health and substance abuse issues, and it is possible that patients in category $\mathrm{A} / \mathrm{B}$ prisons have more of these kinds of needs compared with patients in other prisons, or that their needs can only be met within the prison environment because of security concerns. However, it is not self-evident whether 
male patients in category $\mathrm{C} / \mathrm{D}$ prisons should receive a narrower range of specialised services simply because of their lower-security status.

Women's prisons had relatively high levels of health care support, including both GP and nurse support, even after adjustment for size. Patterns of vacancies cannot explain this result, as the data show that vacancies were more common at women's prisons.

\section{Strengths and limitations of the study}

We obtained a good response rate, and the survey is likely to be representative of prisons as a whole. Prison size was missing for $20 \%$ of units, and data on nurse sessions had to be imputed for a sizable number, making the results for these variables less reliable.

Although the survey methodology is useful in providing a broad overview, further in-depth study of some of the findings would be useful. This would include the extent of the apparent problems with nurse provision.

The survey relies on self-report rather than on observed activity. A key assumption is that health care managers were aware of the full breadth of services being delivered within their prison, which may not be true. The general view is that selfreport tends to over-estimate or over-value available services (Ewing et al., 1999); thus, the true extent of problems may be greater than identified in this survey. Because this was an exploratory study, we used an alpha level of $5 \%$, but recognised that the number of statistical tests conducted is likely to have generated some spurious chance associations.

\section{Implications for future research}

The survey points to the need to investigate more fully why primary care provision for chronic diseases in prisons is likely to be poorer than in the community and to develop effective means to close this gap. Areas of note include the use of IT systems and nurse recruitment and retention, which may act as potential constraints on service development in prisons. Although there is international evidence about primary health care needs of prisoners (Watson et al., 2004), there is, to our knowledge, a lack of evidence about primary health care provision in prisons worldwide; research enabling comparisons to be made would be useful. Although prisoners' views of health care services have been investigated, there is a lack of knowledge about patient self-management of chronic diseases in prison and how best to promote self-care. There is a need also to understand why patients in some types of prisons, notably large prisons and category A/B prisons, appear to have access to a wider range of specialised services than those in other types of prisons.

\section{Acknowledgements}

We are very grateful to the health care managers who undertook to complete the questionnaire. The survey was funded through the Prison Health Research Network.

The survey was essentially a 'self-report audit' of existing prison services by the Department of Health. The investigators were acting as the government's agents in conducting the audit. As such, the project was exempt from the need for Research Governance or Ethics Committee approval.

\section{References}

Balas, E.A., Weingarten, S. et al. 2000: Improving preventive care by prompting physicians. Archives of Internal Medicine 160, 301-08.

Birmingham, L. 2003: The mental health of prisoners. Advances in Psychiatric Treatment 9, 191-201.

Bodenheimer, T., Wagner, E.H. et al. 2002: Improving primary care for patients with chronic illness. JAMA 288, 1775-779.

Boutwell, A.E., Allen, S.A. et al. 2005: Opportunities to address the hepatitis $\mathrm{C}$ epidemic in the correctional setting. Clinical Infectious Diseases 40, (Suppl. 5), S367-372.

Butler, T., Allnutt, S. et al. 2005: Mental disorder in the New South Wales prisoner population. Australian and New Zealand Journal of Psychiatry 39, 407-13.

Butler, T.G., Dolan, K.A. et al. 1997: Hepatitis B and C in New South Wales prisons: prevalence and risk factors. Medical Journal of Australia 166, 127-30.

Butler, T., Kariminia, A. et al. 2004: The self-reported health status of prisoners in New South Wales. Australian and New Zealand Journal of Public Health 28, 344-50.

Campbell, S.M., Hann, M. et al. 2001: Identifying predictors of high quality care in English general practice: observational study. British Medical Journal 323, 784-87.

Condon, L., Hek, G. and Harris, F. 2007: A review of prison health and its implications for primary care nursing in England and Wales: the research evidence. Journal of Clinical Nursing 16, 1201-209. 
Department of Health. 1999: The future organisation of prison health care. London: Joint Prison Service and National Health Service Executive Working Group.

Department of Health and HM Prison Service. 2001: Report of the working group on doctors working in prisons. London: Department of Health.

Department of Health and HM Prison Service. 2002: Developing and modernising primary care in prisons. London: Department of Health.

Department of Health and HM Prison Service. 2006: $A$ twelve-month study of prison healthcare escorts and bedwatches. London: Department of Health.

Department of Health and Home Office. 2007: National partnership agreement between the Department of Health and the Home Office for the accountability and commissioning of health services for prisoners in public sector prisons in England. London: Department of Health.

Docherty, J. 2007: The healthcare challenges of older people in prisons. http://www.phrn.nhs.uk/workstreams/primary care/OlderPrisoners.pdf

Ewing, G.B., Selassie, A., Lopez, C. and McCutcheon, E. 1999: Self-report of delivery of clinical preventive services by US Physicians. American Journal of Preventive Medicine $17,62-72$.

Farrell, M. et al. 1994: Methadone maintenance treatment in opiate dependence: a review. BMJ 309, 997-1001.

Gray, A., Pearce, S. et al. 2006: The training needs of doctors working in English and Welsh prisons: a survey of doctors. International Journal of Prisoner Health 2, 121-30.

Harris, F., Hek, G. et al. 2007: Health needs of prisoners in England and Wales: the implications for prison healthcare of gender, age and ethnicity. Health and Social Care in the Community 15, 56-66.

Macdonald, W. 2006: The health needs of young offenders. http://www.phrn.nhs.uk/Prison/PCSysRevYO.pdf.

MacFarlane, I.A., Gill, G.V. et al. 1992: Diabetes in prison: can good diabetic care be achieved? British Medical Journal 304, 152-55.

Maher, L., Chant, K. et al. 2004: Risk behaviors and antibody hepatitis $\mathrm{B}$ and $\mathrm{C}$ prevalence among injecting drug users in south-western Sydney, Australia. Journal of Gastroenterology and Hepatology 19, 1114-120.
Marteau, D. and Farrell, M. 2005: Clinical management of substance misuse in prisons (Chapter 23). In Gerada, C., editor, RCGP guide to the management of substance misuse in primary care. London: RCGP.

Martin, E., Russell, D. et al. 1991: Patients' views of the consultation: comparison of a prison and general practice population. British Journal of General Practice 41, 207-09.

National Offender Management Service. 2007: Population in Custody Monthly tables April 2007 England and Wales. Ministry of Justice. http://www.justice.gov.uk/docs/ population-in-custody-0407.pdf.

Olubodun, J. 1996: Prison life and the blood pressure of the inmates of a developing community prison. Journal of Human Hypertension 10, 235-38.

Pettinari, C. 1996: The perception, presentation and assessment of illness in prison: a pilot study. Report to Prison Service Directorate of Health Care, Centre for Work, Interaction, and Technology. School of Social Studies, University of Nottingham.

Reed, J. and Lyne, M. 1997: The quality of health care in prison: results of a year's programme of semistructured inspections. BMJ 315, 1420-424.

Singleton, N., Meltzer, H. et al. 1998: Psychiatric morbidity among prisoners in England and Wales: the report of a survey carried out in 1997 by Social Survey Division of the office for National Statistics on behalf of the Department of Health. London: The Stationary Office.

Twaddle, A.C. 1976: Utilization of medical services by a captive population: an analysis of sick call in a state prison. Journal of Health and Social Behavior 17, 236-48.

Watson, R., Stimpson, A. and Hostick, T. 2004: Prison health care: a review of the literature. International Journal of Nursing Studies 41, 119-28.

Weingarten, S.R., Henning, J.M. et al. 2002: Interventions used in disease management programmes for patients with chronic illness - which ones work? Meta-analysis of published reports. BMJ 325, 925-28.

White, P., Park, I. et al. 1999: Prison population brief, England and Wales: March 1999. London: Home Office.

Woodroffe, E. 2006: Nurse-led general practice: the changing face of general practice? British Journal of General Practice 56, 632-33. 